CARNETS DE Carnets de géographes

GÉOGRAPHES.

\title{
Cartographier les émotions
}

\section{Philippe Rekacewicz et Bénédicte Tratnjek}

\section{(2) OpenEdition}

Journals

Édition électronique

URL : http://journals.openedition.org/cdg/687

DOI : $10.4000 /$ cdg. 687

ISSN : 2107-7266

Éditeur

UMR 245 - CESSMA

Référence électronique

Philippe Rekacewicz et Bénédicte Tratnjek, « Cartographier les émotions », Carnets de géographes [En ligne], 9 | 2016, mis en ligne le 20 décembre 2016, consulté le 21 décembre 2020. URL : http:// journals.openedition.org/cdg/687 ; DOI : https://doi.org/10.4000/cdg.687

\section{(c) (1) (9)}

La revue Carnets de géographes est mise à disposition selon les termes de la Licence Creative Commons Attribution - Pas d'Utilisation Commerciale - Pas de Modification 4.0 International. 


\section{CARTOGRAPHIER LES EMOTIONS \\ Entretien avec Philippe Rekacewicz}

Réalisé par Bénédicte Tratnjek, le 22 avril 2016.

Les notes entre crochets, les notes de bas de page et les ajouts de citations sont de B. Tratnjek.

Philippe Rekacewicz est géographe, cartographe et journaliste. II a été un Collaborateur permanent du Monde diplomatique de 1988 à 2014 et chef de projet au PNUE (Programme des Nations unies pour l'Environnement) de 1996 à 2008. II co-anime aujourd'hui - avec Philippe Rivière - le site Visionscarto ${ }^{1}$. C'est I'un des principaux défenseurs de la géographie et de la cartographie critique en France. Philippe Rekacewicz revendique et souligne la subjectivité cartographique en livrant de nombreuses esquisses réalisées à la main. Pour lui, "l'esquisse réhabilite la dimension émotionnelle, d'ordinaire absente de la carte imprimée, froide et scientifique ".

De la carte à la carte en colère : la cartographie critique et les émotions

Bénédicte Tratnjek (BT): Dans votre démarche de cartographie critique, la colère ${ }^{2}$ est prédominante $^{3}$. Pourquoi la colère, et plus généralement les émotions, sont-elles si importantes dans votre travail ?

Philippe Rekacewicz (PR): Une partie de mon travail est de réintroduire les gens dans la carte. Cela suppose de se questionner sur la manière d'appréhender, de capter, de représenter les émotions. II faut d'abord interroger le processus de création de l'image cartographique : le cartographe n'accouche pas d'une image qui serait une retranscription fidèle de la réalité. La carte porte la marque de son auteur, cette marque est une référence, une vision de l'auteur. Or, on considère souvent la carte comme un objet scientifique indiscutable, comme une vérité absolue derrière laquelle l'auteur et son "intention cartographique » disparaît La subjectivité, l'objectivité, la neutralité sont trois concepts différents, et on fait souvent des confusions entre ces trois termes.

\footnotetext{
${ }^{1}$ http://visionscarto.net

2 Rekacewicz, 2013a.

Voir également : "Dessiner le monde (3/4) - Cartes en colère ", Culturesmonde, émission animée par Florian Delorme, avec Philippe Rekacewicz, Khalil Tafakji et Delphine Papin, 26 décembre 2012, audio en ligne: http://blog.mondediplo.net/2013-12-22-Cartes-en-colere

${ }^{3}$ On pourrait par exemple citer la carte des milliers de morts aux portes de l'Europe ${ }^{3}$, réalisée avec le géographe Olivier Clochard
} 
La base de tout ça vient de mon parcours: je ne suis pas géographe ET cartographe par hasard. Une dimension personnelle, un peu traumatique, joue un rôle important dans mon parcours. Cartographe, c'est plus qu'un métier pour moi, c'est une vocation. Ma famille, très cosmopolite, a parcouru toute l'Europe. Elle comprend des membres des quatre religions, des histoires de pogroms, une grand-mère d'une riche famille d'Odessa qui a fui la révolution russe, qui est arrivée à Vienne et a vécu à quelques pas de la maison de Freud et de la famille du pianiste légendaire Rudolf Serkin, dans un quartier d'artistes, son père qui a créé la première maison de réfugiés... L'histoire de ma famille, c'est plusieurs berceaux qui se trouvent dans un espace et un temps particulier: l'Autriche et l'Europe centrale des années 1920 d'un côté, Ellis Island et la communauté russo-polonaise en Pennsylvanie au début du siècle de l'autre.... Et son cortège de mystère. Tout cela a provoqué en moi l'envie d'être très attentif à l'espace et au temps.

C'est ainsi que j'observe le mouvement Nuit debout, qu'il m'interroge, notamment par ses risques de dé-spontanéiser le spontané. Il y a eu la même chose dans le milieu de la cartographie en France, cette volonté de ceux qui possèdent, diffusent et enseignent la cartographie de la contrôler. L'école de Jacques Bertin, en ce sens, est à la fois révolutionnaire (ce n'est pourtant pas le premier à produire un langage sémiologique, cela existe dès le $X I X{ }^{e}$ siècle, mais Bertin en a fait la synthèse, a réorganisé, conceptualisé des pratiques qui existaient mais dans le désordre) et dogmatique. On ne peut plus faire autre chose. Or on peut tellement retreindre un domaine en le dogmatisant...

Ces trois ou quatre dernières décennies, les " cartographes thématiciens " ont répondu à l'actualité avant tout, dans un processus assez aliénant qui n'autorisait que rarement la fantaisie et la créativité. Comme l'artiste Ben qui voulait faire " du nouveau en art " certains géographes et cartpgraphes se sont demandés si on pouvait faire "du nouveau en cartagraphie "... C'est ainsi que sont peu à peu apparus des projets de cartographie expérimentale, de cartographie sensible ou encore de cartographie émotionnelle. Et dès lors, les liens entre l'art et la cartographie devenant évidents, beaucoup d'artistes se sont emparé de la carte soit en l'intégrant dans leur œuvre soit en en faisant leur objet principal. Et c'est dans ce contexte que le FRAC Lorraine (Fond régional pour l'art contemporain) m'a passé commande d'une " œuvre cartogaphique » de 9 mètres de long sur 2 mètres de haut (Figure 1), qui a été exposée entre juin et octobre [2016] ${ }^{4}$.

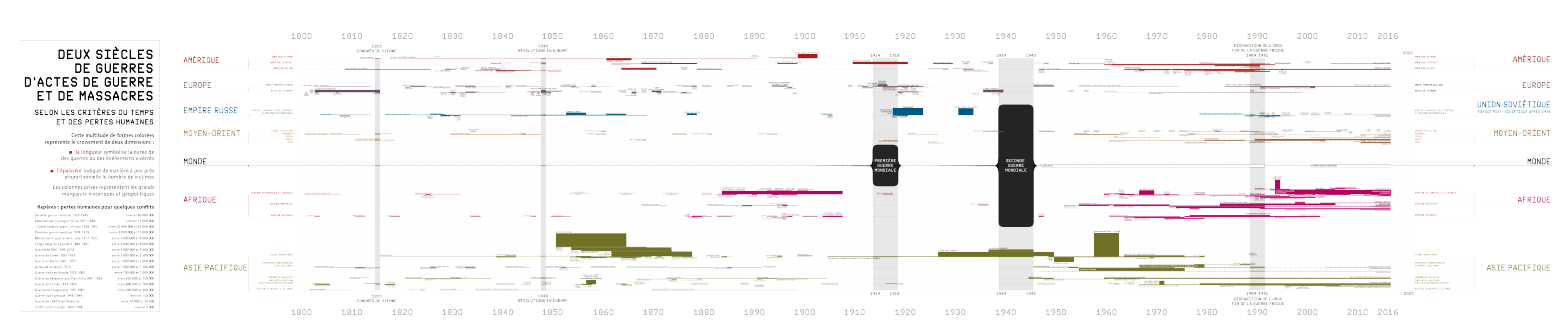

Figure $n^{\circ} 1$ : Deux siècles de guerres ininterrompues

Source: Philippe Rekacewicz, 2016, exposition Zones Sensibles. La carte fait-elle le monde ?, FRAC de Lorraine, Metz, 24 juin/23 octobre 2016.

\footnotetext{
${ }^{4}$ Exposition Zones Sensibles. La carte fait-elle le monde ?, 24 juin/23 octobre 2016, FRAC Lorraine, Metz, informations en ligne : http://www.fraclorraine.org/explorez/artsvisuels/449
} 
On voit là le paradoxe de la cartographie actuelle : elle se dédouble entre ce qui est une discipline encadrée, avec des méthodologies, voire des dogmes d'une part, et la création spontanée, la cartographie qui repose sur la spontanéité et l'expérimentation d'autre part.

Est-il possible de résister à cette tendance de vouloir classer les choses dans des petites cases logiques, systématiquement regrouper ce qui semble se ressembler ? Au musée Léopold de Vienne, par exemple, un panneau indique que toutes les œuvres ont été mélangées! II n'y avait plus de catégories (en termes de styles, périodes ou écoles) pour " ranger " les peintures. Les œuvres ont été réorganisées pour montrer autre chose : les liens entre les formes, les couleurs, les mouvements. Ce choix muséographique original et inédit, est l'expression de ce qu'on aimerait faire en " cartographie expérimentale ». Et c'est un aspect important de ma démarche, mais assez compliqué à mettre en œuvre, car nous aussi, en cartographie expérimentale, utilisons des dogmes et des codes dont il est difficile de se défaire. Sortir de la méthode, d'expérimenter d'autres formes est un véritable défi pour nous du point de vue de l'acte de création, toujours anxiogène, mais aussi pour le transmettre au public, aux lecteurs sans les déboussoler. Nous ne pouvons pas nous lancer dans des projets complètement abstraits, car il faut garder le contact avec un public qui doit pouvoir comprendre ce que nous proposons. Nos créations cartographiques sont produites dans un cadre social, pour un public qui doit pouvoir les comprendre, les utiliser, les discuter.

BT : Vous abordez la question des liens entre la carte et les arts. Comment replacer la cartographie dans le contexte artistique? Quels sont les liens entre arts, cartographie, pouvoir et émotions?

PR : Les cartes anciennes relevaient du secret d'Etat : pour asseoir son pouvoir, il faut avoir la carte. Cela me fait penser au roman historique Le Planisphère d'Alberto Cantino. Lisbonne, 1502 (Gérard Vindt, 2000), une histoire basée sur un réalisme historique : le souverain envoie ses navigateurs lever la carte de son empire pour qu'il puisse y exercer son plein contrôle. Mais un jour dans le cabinet cartographique royal, la carte - qui était un exemplaire unique - disparaît... et avec la carte disparaît aussi le pouvoir du roi qui n'a plus l'instrument pour diriger son empire. Cette histoire est un puissant symbole du pouvoir de la carte.

On peut prendre un exemple concret : une carte du transport de pétrole, une représentation très classique, que l'on voit partout, qui donne à voir la production et la consommation de pétrole à l'échelle du monde. C'est une carte qui décrit un événement réel et connu. La carte se fait ici témoin d'une situation qui s'inscrit dans le monde du visible. Mais qui sait que ce pétrole brut peut-être revendu et acheté plusieurs fois pendant le voyage dans un mouvement spéculatif au gré des changements des cours ? II faudrait alors inventer la carte qui montrerait cette situation " invisible " à l'œil nu... Cette complexité doit faire face à une double difficulté: établir les faits et les données, puis en donner une images carto(graphique). C'est bien ici que la cartographie expérimentale a toute sa place: l'immense travail de collecte de données n'est pas suffisant. Une carte conventionnelle n'apporterait pas beaucoup plus que les données brutes. 
Croiser l'art et la cartographie est une manière d'expérimenter. Cela me fait penser au travail de la géographe Elise Olmedo [voir par exemple : Olmedo, 2011, 2015a et 2015b; Olmedo, Poisson, 2012 ; Olmedo, Roux, 2014a et 2014b ; Feidel, Olmedo et al., 2016]. Elle a suivi des femmes d'un quartier défavorisé à Marrakech pour essayer de comprendre et cartographier leur déplacement dans la ville, leur utilisation de l'espace public ainsi que dans la sphère privée. Au début de ses recherches, elle a produit beaucoup de cartes conventionnelles, mais qu'elle jugeait elle même indigentes et très " pauvres » au regard de la force de son sujet. Elle a donc cherché - et trouvé - un autre moyen de représenter la manière dont ces femmes se projetaient dans leur espace de vie leur appréhension de l'espace public, etc. Une question majeure se posait : comment représenter leurs émotions ? La cartographie expérimentale lui a permis de trouver des moyens beaucoup plus adaptés, beaucoup plus fort pour représenter les émotions, le ressenti [Olmedo, 2011]. Elise Olmedo s'est projetée dans la carte, mais les vrais " héros" de sa carte sont les femmes qui l'ont coproduites avec elles, et qui y parlent de leurs émotions, de leur rapport sensible à l'espace.

On peut aussi prendre en exemple ce qui se passe dans les terminaux des aéroports, comment s'organisent les infrastructures et la circulation. Quand on est sur place, sur le terrain, on regarde, on observe, on s'imprègne de l'atmosphère pour comprendre et " déconstruire " la logique d'organisation de l'espace (souvent invisible) pour finalement le reconstruire sur la carte en montrant, en expliquant les processus en cours (et donc les rendre visible pour le public). Ce qui revient à dire que nous reconstruisons un nouveau discours, naturellement très éloigné de la narration officielle ou conventionnelle. Le lent grignotage de l'espace public par le commerce dans les aéroports et la suppression progressive des couloirs publics, imposent aux usagers les "espaces marchands ". Cela change notre rapport à l'espace: "dans cet espace "du dedans ", tout est restreint, de la liberté de se regrouper à celle de photographier ou de filmer " [Rekacewicz, 2013b]. L'aéroport n'est plus un espace de transit, il est un espace de consommation qui provoque le dégoût, et il faut traduire cela en carte : traduire un sentiment, et donc réhabiliter l'émotion cartographique (Figure 2). 
Figure 2 : L'aéroport d'Oslo et les transformations de l'espace en 2005 et 2013

ULTRA MODERAE ET CONFORTABLE,

ILY FAISAT BON SE POSER DANS LE CAMHE ET LE SILENCE

POUR OUBLIER LE CHAHTT DU DEHORS
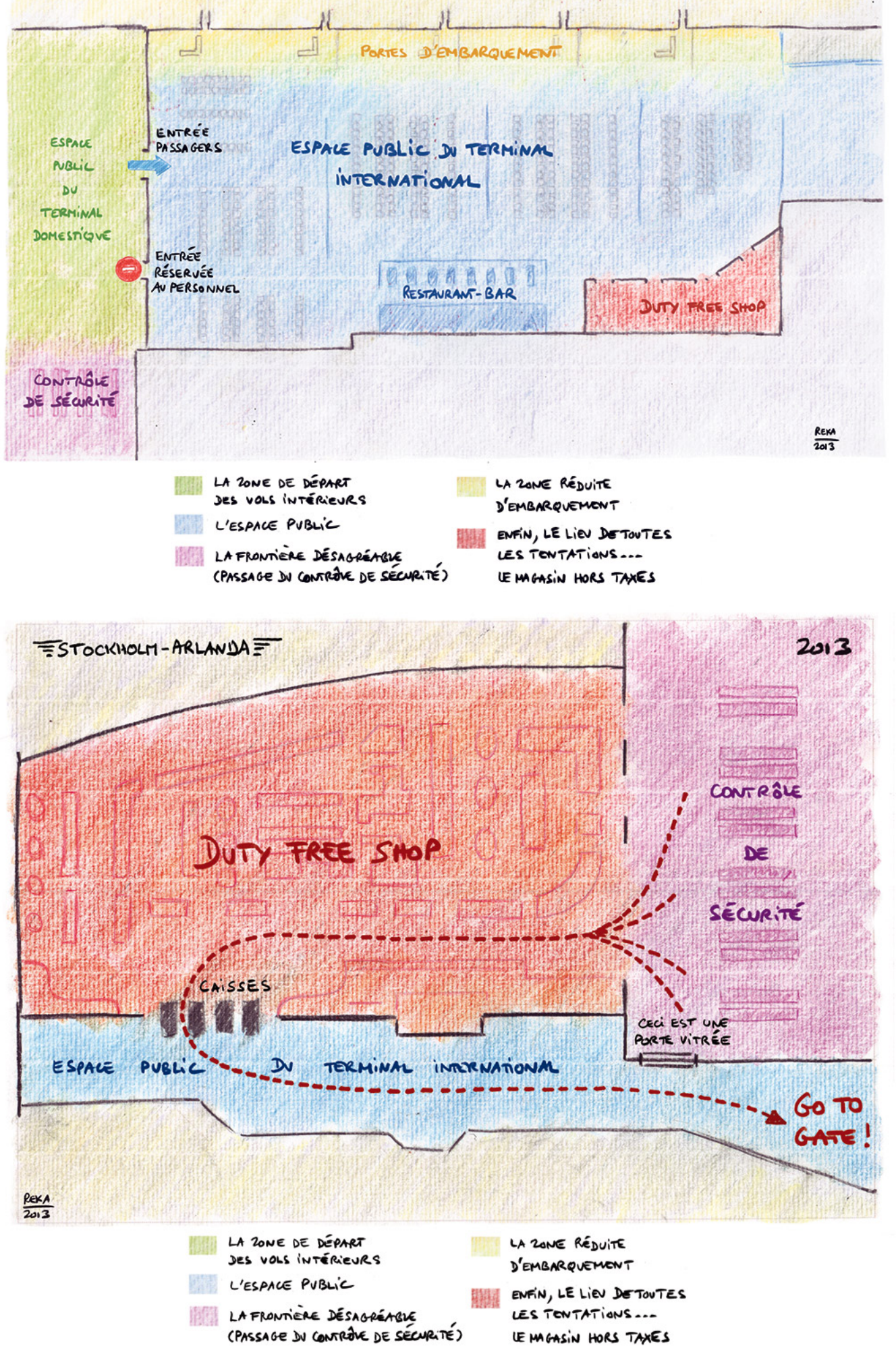

Source : Rekacewicz, 2013b. 


\section{Cartographie radicale et émotions: réintroduire l'émotion cartographique, réhabiliter l'imprécision géographique, oser l'esquisse}

BT : Vous parlez beaucoup des cartes sensibles. Quelles différences peut-on établir entre cartographie émotionnelle (telle que pratiquée par Christian Nold ${ }^{5}$ ) et cartographie sensible (telle que pratiquée par Elise Olmedo)? Peut-on ou doit-on établir une différence?

PR : Les deux permettent la réintroduction de l'émotion dans la cartographie, et c'est un point qui me semble fondamental. A mon sens, l'esquisse permet de réhabiliter l'émotion cartographique, totalement absente des cartes de plus en plus techniques. Je me permets de prendre plusieurs exemples.

La carte des "morts par milliers aux portes de l'Europe " [Clochard, Rekacewicz, 2004] est l'une des premières séries d'esquisses, présentée au Musée d'art moderne à Vienne (Figure 3). Tout est parti d'un lieu emblématique situé au cœur du district de Leopoldstadt, à Vienne : le magasin de Sharon Stone, commerçante ghanéenne.

C'est probablement la seule façade de magasin vert pomme en Autriche. Impossible de la manquer. Sharon y vend des produits de beauté, des épices et un peu d'alimentation (dont du poisson congelé), des perruques et des produits pour les cheveux - d'ailleurs, dans un coin, quelques sièges et quelques miroirs font office de salon de coiffure. Enfin, des sofas confortables s'offrent aux clients qui doivent attendre. Sharon est originaire du Ghana et se définit elle-même comme une "business woman". Sa boutique est devenue, au fil des ans, un des points de rencontre les plus importants pour les communautés africaines de Vienne. (Rekacewicz, 2007a).

Sharon est commerçante, et son magasin est devenu - un peu malgré elle - un centre de ralliement pour la vie culturelle et sociale africaine à Vienne. Dans un projet artistique initié par le musée, des artistes sont partis de ce lieu pour explorer toutes les dimensions possibles des liens qui unissaient l'Autriche à l'Afrique. Et de cette exploration participative est née l'exposition « Rendez-vous à Sharon Stone ».

Revenons à la carte " Mourir aux portes de l'Europe », aujourd'hui très connue et reprise. La carte initialement produite dans laquelle les décès de migrants étaient figurés par des centaines de points, établis et localisés scientifiquement, présenté en une typologie assez précise. J'ai finalement décidé de simplifier la carte et de regrouper cette multitude de points en quelques cercles proportionnels au nombre de décès par lieu (Détroit de Gibraltar, Lampedusa, etc.) pour finalement obtenir - et ce fût une surprise - un résultat effrayant : l'esquisse montrait la frontière européenne, c'est-à-dire les limites de la zone Schengen, comme étant sans aucun doute une des plus mortelles au monde (33000 morts entre 1994 et 2016, estimation a minima...).

Lors du vernissage de l'exposition, deux réfugiés nigérians se sont longuement arrêtés devant cette carte. Et après quelques minutes d'un long silence, l'un deux dit, en regardant ces ronds rouges nerveusement crayonnés : "On voit bien ici que le cartographe était très

\footnotetext{
${ }^{5}$ On peut retrouver les travaux de Christian Nold sur plusieurs sites Internet, notamment :

- $\quad$ Le site Christian Nold, http://www.christiannold.com,

- Le site Emotional Cartography, http://emotionalcartography.net,

- $\quad$ Le site Bio Mapping / Emotion Mapping, http://www.biomapping.net.
} 
en colère quand il a dessiné cette carte ". J'ai été assez touché, parce que c'était bien là l'objectif : réintroduire l'émotion dans la production cartographique. C'est d'ailleurs grâce à cette réflexion très touchante que l'exposition cartographique suivante, présentée à la Maison des Métallos à Paris en 2012, s'est appelé, Cartes en colère. Cette colère, en réalité c'était la symbiose entre leurs émotions et la mienne. Lors de la présentation de l'exposition Frontières, réfugiés, migrants ${ }^{6}$ en mars 2007 au Mans, j'ai été très ému lorsque j'ai vu des visiteurs sortir en larmes. C'est peut-être là que se joue le passage de la carte informative à la carte émotionnelle.

Figure 3 : Installation cartographique dans la grande salle de la Kunsthalle Exnergasse en novembre 2007

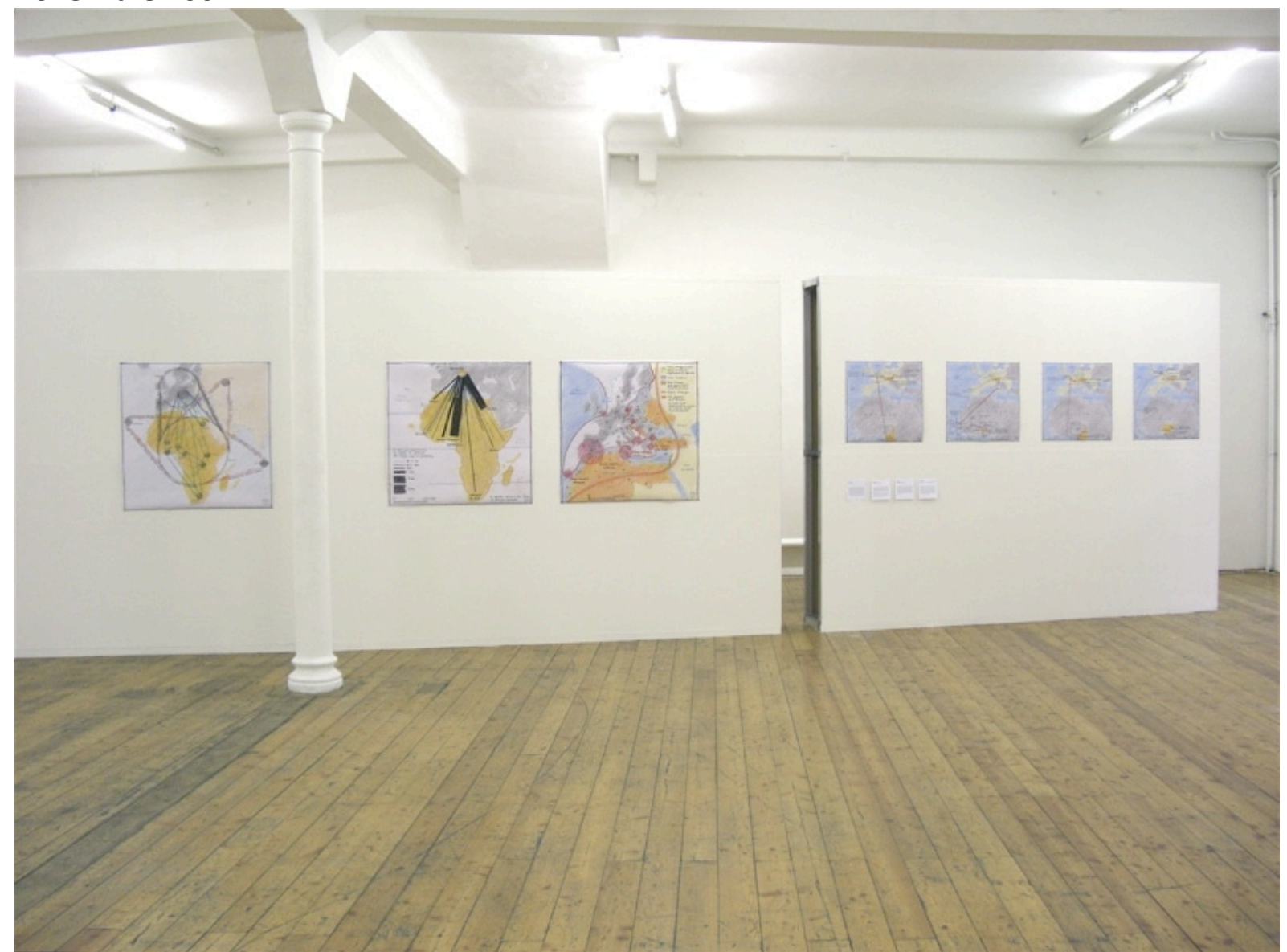

Source : Rekacewicz, 2007a.

La deuxième intention, après la réintroduction de l'émotion, est la réhabilitation de l'imprécision géographique. J'avais l'intuition que, dans notre démarche de thématicien, la précision n'était pas si importante. L'esquisse cartographique me permet de montrer les grands mouvements, les grandes zones, les grandes tendances. Ce qui est souvent suffisant pour poser les termes du débat ! Je me suis accroché à cette idée, mais l'idée n'est pas de rendre la carte encore plus approximative (ou floue), mais de montrer, de rendre visible en quoi elle est justement approximative.

\footnotetext{
${ }^{6}$ A ce propos, voir : Rekacewicz, 2006a, 2006b, 2006c, 2006d, 2006e, 2006f, 2007b. Voir également Comité international de Bologne pour la cartographie et l'analyse du monde contemporain, 2008.
} 
L'esquisse est également une manière de distancier la volonté paranoïaque de certains cartographes de faire de la précision à tout prix. Très souvent, dans les phénomènes que nous cartographions, il y a de l'imprécision. Or celle-ci est très difficile à représenter sur des logiciels informatiques. L'esquisse permet si l'on peut dire de reproduire fidèlement l'imprécision, et la rend acceptable. Imaginons que je veuille montrer que les investissements allemands se concentrent dans la région de Moscou et que l'Allemagne a le regard tourné vers l'Est. Sur l'esquisse, je peux représenter cette idée; or c'est quasi impossible de faire ce dégradé sur mon logiciel : ils sont trop nets, le gradient est parfait. Or ce qu'on veut montrer, c'est que c'est discontinu dans l'espace! (Rekacewicz, dans Casanova, Izambert, 2010).

Prenons l'exemple de l'élaboration d'une carte des conflits à l'échelle du monde. Le service minimum serait de "seulement " séparer les pays en guerre des pays en paix. Mais il y a toute une variété de situation, de statut, très difficile à appréhender et donc à cartographier. Un pays comme le Tchad, par exemple, voisin de nombreux territoires en guerre (Soudan/Darfour, République centrafricaine) se trouve lui aussi affecté, et même s'il n'y a pas de combats, il devient un pays à risque, un pays " déstabilisé ". C'est le cas aussi pour la Jordanie, un État en paix dans un environnement régional en guerre (Syrie, Israël/Palestine) : Avec le poids des réfugiés, et la volatilité de la situation régionale, il me semble pertinent de figurer ce pays en zone à risque combien même la situation y est relativement stable. Un pays voisin d'au moins deux pays en guerre est un pays "à risques " même s'il est en paix. On revient à cette idée de zones, et de grandes tendances. C'est ainsi que je construis les cartes globales, en recherchant les grandes tendances, en regroupant les zones, en pensant autrement que par des « cases » colorées à l'intérieur des frontières interétatiques.

Pour moi, la carte n'est jamais l'expression d'une vérité ou d'une réalité indiscutable, mais plutôt d'une intention et $d^{\prime}$ 'une construction intellectuelle soigneusement élaborée qui posent les termes d'un débat, qui est une base de discussion.

Mais sur ces cartes représentant la guerre, il manque souvent des acteurs: on a bien toujours les zones de conflits et les réfugiés, mais moins souvent les interventions militaires extérieures, celles qui viennent de loin (Etats-Unis, France, Russie), et encore moins souvent la figuration des producteurs et exportateurs d'armes. Ils font pourtant partie intégrante du paysage de la guerre dans le monde, et à ce titre il est important de les réintroduire sur la carte.

BT : Le fait de proposer au public des esquisses cartographiques, est-il, pour vous, un moyen de réintroduire cette " émotion cartographique " ?

PR : L'esquisse est une forme graphique qui est à la fois cartographique et artistique, mais qui est aussi temporaire. On retrouve là le projet de la cartographie expérimentale, qui va chercher chez les artistes d'autres manières de représenter un monde en mouvement. Malgré l'actualisation des données, la carte est toujours temporaire, et l'esquisse ne cache pas cet aspect éphémère, qu'elle assume pleinement, elle n'a donc pas besoin d'une réactualisation.

L'esquisse permet de proposer des visions cartographiques à un moment précis, pour garder la mémoire d'une perception fortement ancrée dans ce temps. L'esquisse est aussi un outil contre l'oubli de ce monde qui va très, très vite. "L'esquisse est [aussi] une manière d'assumer la subjectivité » [Rekacewicz, dans Casanova, Izambert, 2010]. J'avais aussi envie, avec l'esquisse, de réhabiliter la dimension émotionnelle. "Comme dans un musée, la carte peut produire une émotion. Du coup, quand elle est froidement conçue, ce n'est pas possible. 
[...] L'esquisse permet d'atteindre un public nouveau, un public qui n'est pas familier avec les cartes. » [Rekacewicz, dans Casanova, Izambert, 2010].

Les démarches intellectuelles et artistiques sont finalement similaires à celle de la recherche : on part d'intuitions, confirmées ou infirmées au rythme des recherches. On n'est jamais sûr que ce soit une bonne direction, il faut aller et venir, se tromper, reprendre... Pour créer une carte, on part d'une idée, on a une " intention ", qu'on essaye de formaliser sous forme de dessin et c'est pour cette raison qu'elle offre bien une image assumée en tant que vision personnelle du producteur de la carte (le cartographe ou éventuellement son commanditaire). La carte n'est pas une fin en soi, mais plutôt un début en soi, je l'espère toujours être la base d'une réflexion partagée, et non pas une réflexion imposée. L'idée du site Visionscarto.net ${ }^{7}$ est précisément de produire et partager la connaissance et d'ouvrir le débat.

BT : Que ce soit par vos cartes ou sur votre site Internet, l'idée est donc de faire participer le public, ou pour le dire autrement de réintroduire les individus dans les cartes?

PR : C'est l'une de mes envies: ré-impliquer les gens. II existe un grand écart entre la carte thématique et le public, parce qu'on a toujours du mal a s'identifier aux cartes, souvent impersonnelles. Je trouve problématique que les gens ne fassent pas « partie de la carte ».

L'ONG United Against Racism (une fédération d'associations et d'ONG du pourtour méditerranéen ${ }^{8}$ ) a, par exemple, eu l'idée, il y a presque vingt ans, de produire un tableau ${ }^{9}$ qui recense les décès des migrants en route pour l'Europe, avec leur noms, leur origine et leur activité professionnelle (à chaque fois que c'était possible. L'idée était de "repersonnaliser " les morts aux frontières de Schengen, de montrer que derrière les froides statistiques, derrière chaque point sur la carte des « morts par milliers aux portes de l'Europe ", il y a un destin, une famille détruite, un drame humain considérable. C'est une manière de réintroduire dans la carte certains acteurs qui ont été par le passé trop vite remplacé par des chiffres. C'est aussi le combat de certains peuples autochtones 'Amérique du Nord, comme les Zunis par exemple, de se lancer dans des projets cartographiques dont le but est de se " reprojeter » sur la carte d'où ils/elles avaient été expulsé.e.s par le pouvoir colonial.

La cartographie expérimentale telle que je la pense, c'est en priorité de réintroduire les gens dans la carte, à l'échelle du quartier, de la ville, de la région, du pays, du continent, ou même du monde. Je n'arrive pas à m'expliquer pourquoi, depuis si longtemps, on les a écartés des représentations cartographiques. En 1989, pour une exposition à la Cité des sciences de Villette (qui venait juste d'ouvrir), j'ai suivi trois familles pendant plusieurs semaines dans leurs déplacements quotidiens. L'idée était voir si on pouvait cartographier la manière dont elles utilisent l'espace. C'est l'un des premiers projets de cartographie expérimentale: figurer sur la carte là où je vais, tous les jours, une fois par semaine, une fois par mois, là où je ne vais jamais parce que j'ai peur, les lieux où j'aime me perdre et rêver, etc. Chaque famille s'est petit à petit projetée sur la carte, et en regardant le résultat, ont pris conscience avec une grande surprise de leur pratique spatiale, de leur "étendue territoriale ", de la manière dont ils sous-utilisent généralement l'espace, des routines, toujours les mêmes

\footnotetext{
${ }^{7}$ http://visionscarto.net

${ }^{8} \mathrm{http://www.unitedagainstracism.org}$

9 http://www.unitedagainstracism.org/publications/list-of-deaths-card-2/
} 
qu'ils répètent jour après jour, semaine après semaine, et aussi et surtout combien leurs émotions participent de leur manière de pratiquer l'espace.

Lors d'un atelier de cartographie critique à l'Institut Goldsmiths de Londres qui réunissait des étudiants venus du monde entier, j'ai été très frappé par un dialogue entre une architecte palestinienne et un géographe israélien, discutant de leur manière de voir le monde : dans leur univers aussi bouleversé, ils parlent et représentent le monde avec leurs trippes ! Cette démarche permet la réhabilitation de l'émotion, du sensible, dans la manière de représenter l'espace. C'est aussi une manière de réconcilier les citoyens avec leur espace, avec leur territoire, de leur donner un outil qui va leur permettre de comprendre comment ils fonctionnent dans l'espace. C'est enfin offrir aux gens un outil, une méthodologie (avec un corpus donné aux participants des ateliers) pour faire dialoguer l'espace de vie avec la carte.

Dans ce type d'ateliers, je considère que le résultat n'est jamais un résultat « final »: je fais toujours produire des esquisses, qui sont et restent des "chantiers " en cours, susceptible d'évoluer, d'être mis à jour. La diversité des participants est aussi l'occasion de travailler sur le "décentrage" de nos visions, de sortir de nos visions occidentalo-centrées. En cartographie, il existe beaucoup de tentatives de décentrer la carte, mais souvent cela reste très froid, très distant, très technologique : ce sont des représentations qui ne disent rien de la perception des habitants. Lorsqu'une participante d'origine nord-coréenne (mais qui réside au Japon) produit sa vision du monde, on obtient un résultat totalement inédit, une sorte d'ovni cartographique jamais vu ailleurs ! Et c'est justement ce qui est passionnant : on part à la découverte du monde, et on découvre en même temps de nouvelles « visions du monde ". Ces ateliers [de cartographie expérimentales] ont pour vocation de devenir des transmetteur de méthodes et de savoir, pour donner la parole aux gens, pour qu'ils puissent acquérir les moyens d'exprimer leur sensibilité politique et artistique, pour leur ouvrir de nouveaux champs d'expression.

D’où le projet "Le monde vu de... " [Rekacewicz, 2013a], collection de cartes qui représentent la perception de la Pologne par les Polonais, de I'Iran par les Iraniens, de I'Allemagne par les Allemands, etc. Les esquisses cartographiques ont été réalisées à partir d'entretiens menés avec un certain nombre de personnes, entre quatre et six par carte, et pour certaines, sur place (Berlin par exemple). La carte "L'Europe vue de Varsovie » (Figure 4), représentation de la projection polonaise en Europe, montre l'importance toujours très forte de deux immenses traumatismes historiques. Exprimer, concrètement sur la carte, les peurs, les fantasmes ou les traumatismes en plus des réalités géopolitiques " tangibles », enrichit d'autant le dialogue géographique. 
Figure 4 : Le monde vu de Varsovie : un rêve, deux cauchemars

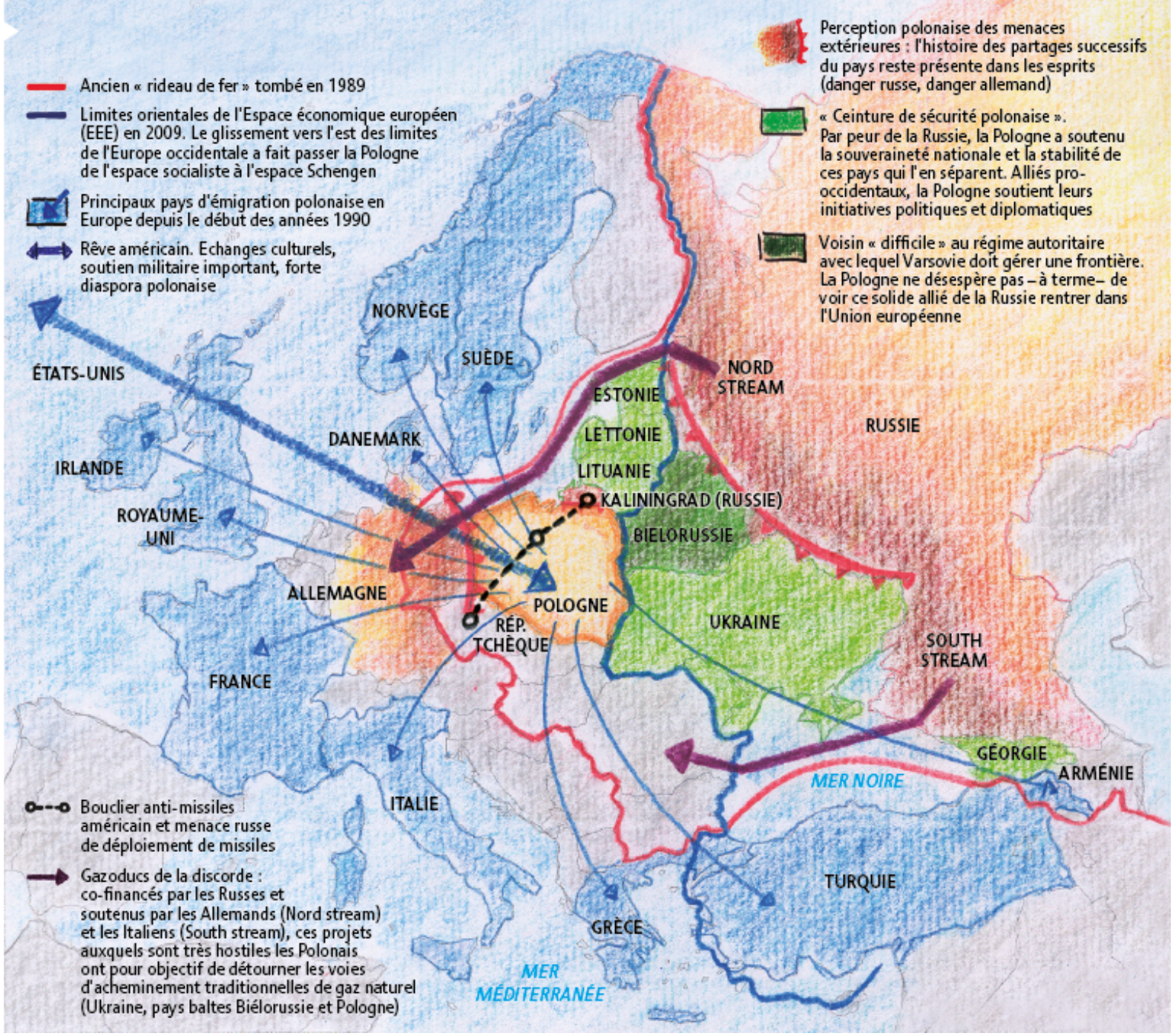

Source : Rekacewicz, 2013a.

Ce sont aussi des cartes idéologiques et même traumatiques qui évoluent (avec, par exemple, la radicalisation de certaines sociétés). C'est pourquoi elles ne doivent pas être finalisées, figées. Ce sont, également, des cartes qui représentent des territoires rêvés, des espaces rêvés. Ces espaces rêvés font partie de ces " mondes vus de ". Par exemple, pour un Hongrois, son "vrai " territoire est aussi en partie en Roumanie, raison pour laquelle Budapest accorde volontiers la nationalité aux Hongrois de Transylvanie. Par conséquent, créer de telles esquisses cartographiques, c'est aussi une manière de réintroduire les gens sur la carte par le biais de leur imaginaire. Cela permet aussi de rendre les gens propriétaires de leur propre vision. Ce ne sont pas seulement des cartes mentales comme outil pour comprendre l'espace, il s'agit de les restituer aux gens, aux acteurs, la propriété de leur vision et de leur image. 
BT : Les émotions des habitants, des acteurs ne sont pas les seules à être en jeu dans la cartographie radicale: qu'en est-il des émotions du cartographe? Pourquoi rendre, contrairement à la tendance générale, de plus en plus présent le cartographe et ses émotions dans la carte?

PR : Entre le producteur de la carte et la carte elle-même, il existe un gouffre énorme. Dans ce gouffre, on trouve : les données, le matériel pour faire la carte, la culture, la formation, l'éthique, les valeurs morales, la sensibilité artistique, etc., c'est-à-dire beaucoup de filtres ! Le cartographe (ou le concepteur de la carte) est, en fait, très loin de la carte.

L'une des idées de la cartographie expérimentale est de réduire sensiblement la longueur de chemin qui mène le concepteur à la carte. La carte n'est pas qu'un objet scientifique créé à partir de chiffres ou d'informations qualitatives et de méthodes pour traiter ces données (méthodes, d'ailleurs, qui ont permis de trouver des voies pour rendre visible l'invisible, et il ne s'agit pas de tout de les disqualifier !). Mais, ces méthodologies, qui ont été utiles, nous ont aussi éloignées de la carte, nous ont déconnectés d'une certaine réalité sur le terrain, de la perception que l'on pouvait avoir.

J'aspire à un lien beaucoup plus direct avec la carte. Commencer directement par la recherche des données peut facilement inhiber la réflexion sur le sens de la carte (puisqu'on est accaparé plus par le travail sur la pertinence des chiffres et des infos que sur le message qu'on voudrait diffuser). Il faut sans doute prendre les choses à l'envers et peut-être tout de suite commencer par jeter sur le papier ses idées, pour être plus proche de l'intention cartographique, puis confirmer/infirmer par les données, et enfin travailler sur les formes, le design, l'esthétique,

Deux exemples emblématiques de cette démarche (bien plus qu'une méthode, il s'agit d'une démarche) : "la sanctuarisation du monde » [Rekacewicz, 2014a], et le monde vu du Japon [Rekacewicz, 2013a].

Dire que je produis des « cartes en colère » (Rekacewicz, 2013a), c'est dire que l'émotion est un moteur pour le cartographe. Soit on est géographe mais "seulement " comme témoin de l'évolution du monde avec les données recueillies; soit on est aussi un citoyen impliqué. Et c'est ici que monte la colère du cartographe, quand on perçoit les injustices. II y a plusieurs manières de répondre à cette colère : devenir activiste, par exemple, ou utiliser les compétence qui sont les nôtres Faire des "cartes en colère " est le déclic qui permet de transgresser les conventions cartographiques traditionnelles. La colère va permettre de matérialiser, par la carte, ce qui indigne le cartographe. Pour moi, c'est notamment de comprendre que les politiques européennes de fermeture des frontières et de restrictions migratoires depuis les années 1990 (basés sur une perception (fausse) d'invasions...) sont directement responsables de la mort de dizaine de milliers de migrants. Cette colère, je l'ai partagée avec Olivier Clochard, et cela a donné la carte " mourir aux portes de l'Europe » [Clochard, Rekacewicz, 2006].

Le monde migrant est très diversifié : l'attention médiatique et politique se porte sur les migrants économiques. Mais il y a d'autres migrants: certains ont un besoin vital de voyager, comme ce Sénégalais atteint du Sida, qui a un urgent besoin de thérapie uniquement disponible en Europe, qui a les moyens de couvrir financièrement son séjour, mais à qui on refuse le visa d'entrée... Et qui se voit obligé de tenter le voyage par les voies les plus dangereuses. Et qu'en est-il de ceux qui sont persécutés dans leur pays pour leur 
appartenance à une minorité, pour leurs pratiques sexuelles ? Et comment transmettre le témoignage de cette colère ?

$C^{\prime}$ est pour faire passer cette colère que j'ai choisi de publier ces esquisses cartographiques, brutales, simplifiées sans être simplificatrices, très directes dans leur message [Rekacewicz, 2014 a et $2014 b]$. Je voulais faire passer la colère par la carte, la partager par la carte. C'est une transgression du langage de la cartographie traditionnelle: rendre légitime par l'émotion, des représentations cartographiques considérées illégitimes ce qu'il est convenu d'appeler les « règles de sémiologie graphie » ou les « dogmes cartographiques ».

Dans la pratique, il s'agit, par exemple, de mélanger sur une même carte les flux touristiques et les flux de migrants. Ne pas séparer les deux thèmes, les montrer ensemble pour rendre visible ce qui se joue, par exemple, un carambolage violent sur une plage des Canaries de deux sociétés qui, de part et d'autre de la Méditerranée, se croisent toujours sans jamais se rencontrer: migrants et touristes. L'origine de cette esquisse [figure 5], c'est une photographie qui représente une jeune touriste en maillot de bain qui recueille et réconforte un migrant subsaharien exténué, arrivé à la nage après que son embarcation eut coulée... De ce croisement de deux destins, il ne faut pas seulement en garder la photographie : il fallait aussi en faire une carte [Rekacewicz, 2009 et 2014b].

\section{Figure 5 : Flux touristiques et flux migratoires en Méditerranée : croisements improbables}
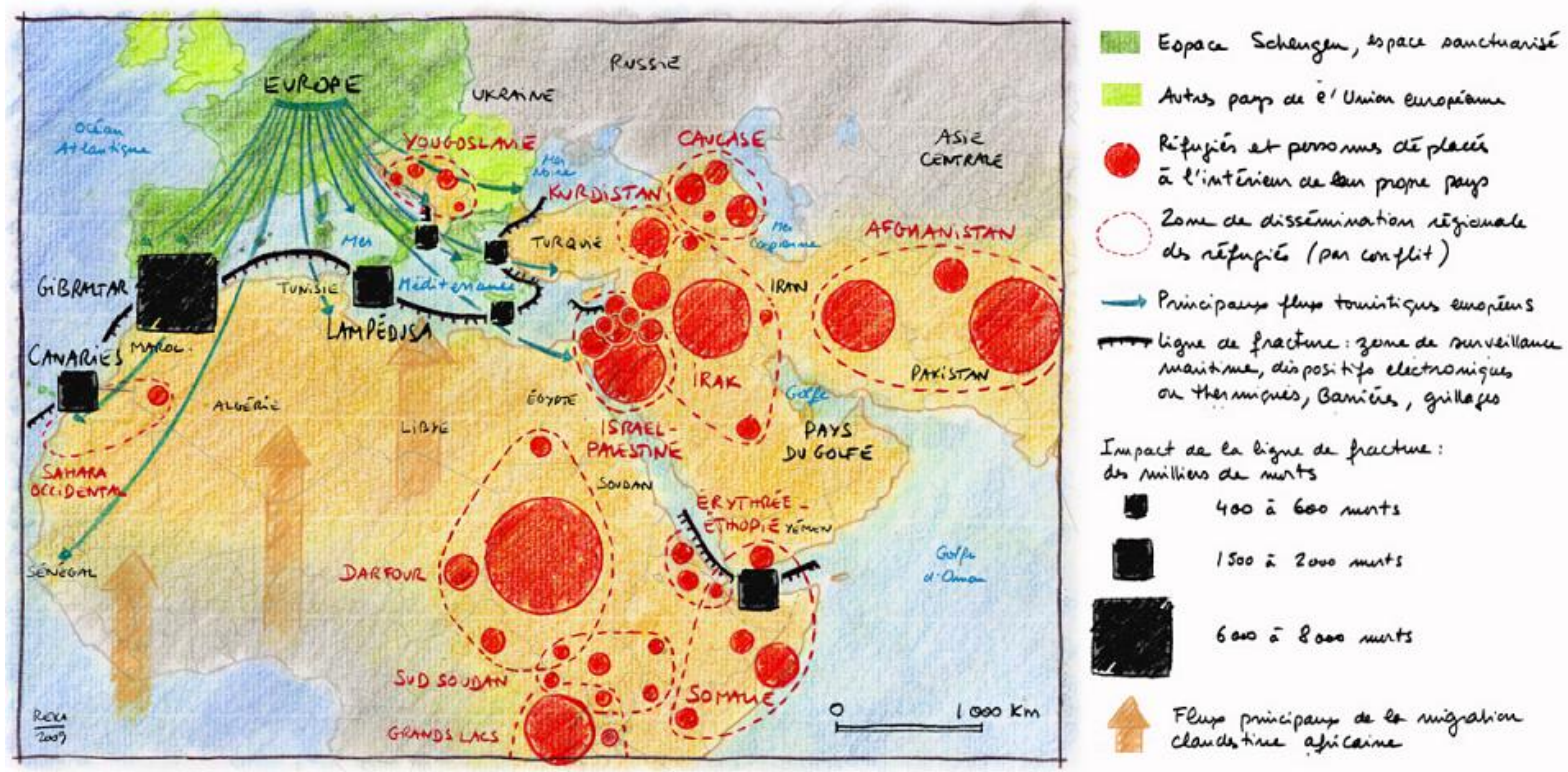

Source : Rekacewicz, 2009.

Le choix des mots dans la carte (le titre, la légende, les toponymes) est aussi un moyen pour le cartographe d'exprimer cette colère. II faut refuser une terminologie susceptible de " criminaliser " cette forme de migration ("flux illégaux ", " migrants clandestins »), et peut-être leur préférer des termes plus humains ("flux irréguliers » ou "informels ", ( " migrants-voyageurs »). le travail de légende et le choix de la terminologie est absolument fondamental dans la démarche expérimentale, en ce qu'il fait partie intégrante de ce travail sur l'émotion, la sensibilité. 
Ma colère de cartographe se focalise aussi sur le fonctionnement de la haute finance. Comment formaliser le gouffre qui séparer les riches et les pauvres ? C'est l'idée du projet en cours « Géographie du vide, géographie du plein » [Rekacewicz, 2013a] (Figure 6) : mettre en évidence l'immensité obscène de ces inégalités.

\section{Figure 6 : Aux marges externes de la légalité}

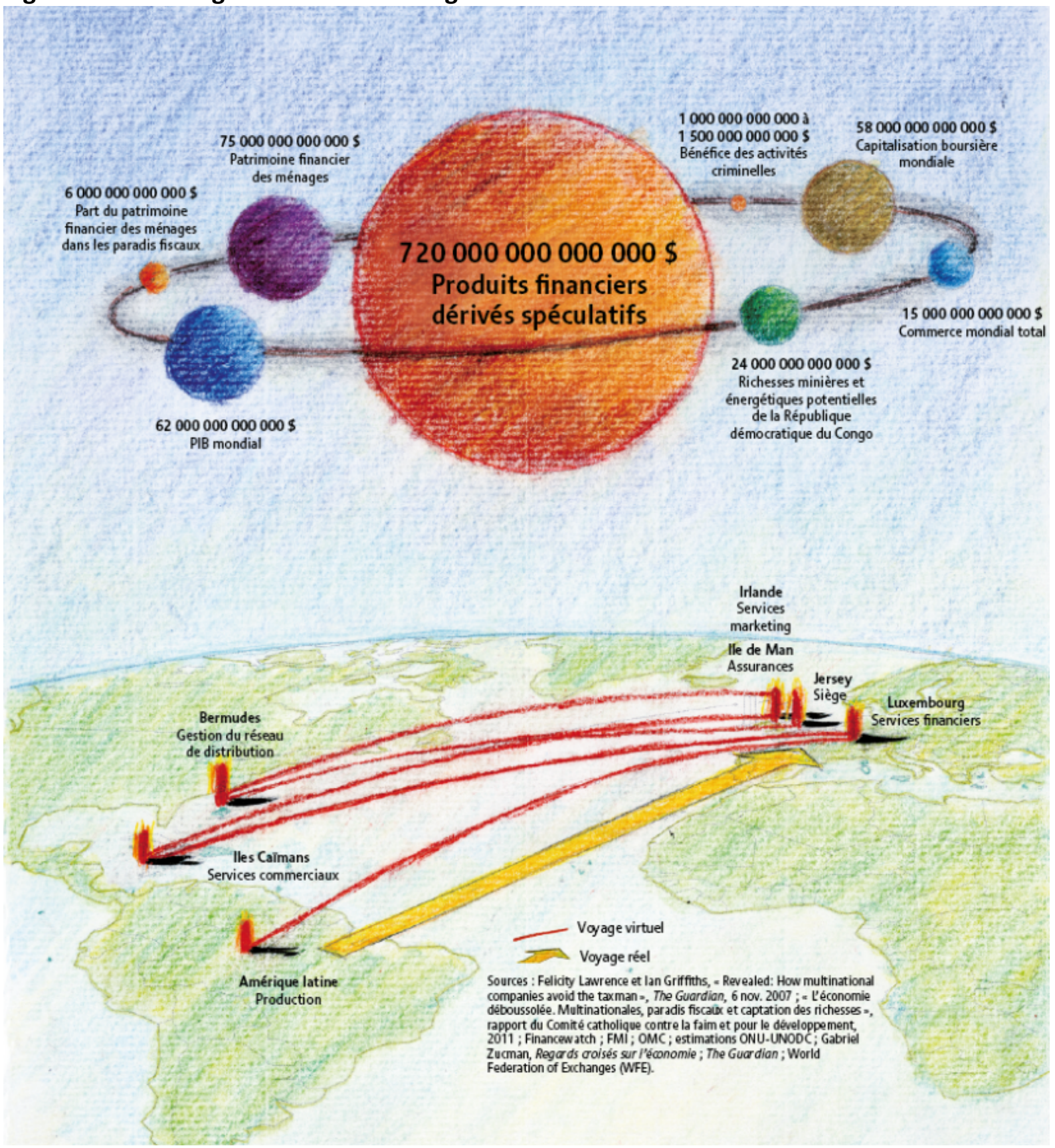

Source : « Mondes émergents ", Atlas 2012 du Monde diplomatique ; Rekacewicz, 2013a.

BT : Vos cartes abordent beaucoup le thème de la violence (la guerre, les morts des réfugiés, la violence du capitalisme et des inégalités, etc.). Comment le cartographe qui veut rendre ses émotions sur la carte gère-t-il la question de la violence dans la carte ? 
N'est-ce pas problématique que la carte ait une dimension esthétique quand elle traite de tels sujets?

PR : Est-ce légitime de faire de la carte une œuvre d'art ? Ça pose éventuellement un problème, surtout quand elle évoque des situations d'une extrême brutalité. Au cours des deux ou trois derniers siècles, la carte a souvent outil pour assoir le pouvoir, consolider la puissance [voir notamment: Rekacewicz, 2006g]. C'était un outil qui permettait d'imposer sa vision du monde, souvent violente d'ailleurs, souvent dans le dénie des peuples. Mais, en même temps, les cartes ont été de vraies œuvres d'art, comme par exemple les portulans, ces cartes marines médiévales européennes qui étaient, pour le cartographe, le projet de sa vie [sur ce sujet, voir notamment : Grataloup, Salachas, Sarazin, 2013].

Le grand paradoxe de la cartographie aujourd'hui est de représenter en même temps du sang, des larmes, de la souffrance, avec une certaine recherche esthétique. Et c'est gênant. C'est pourquoi en cartographiant les guerres, par exemple, j'ai toujours cherche à être efficace, c'est-à-dire, à transmettre correctement ms intentions cartographiques, sans nécessairement rechercher à " faire beau » : je veux une image de mémoire, pas une image esthétique. Les modes de représentation de la violence dans le monde qui nous entoure sont une préoccupation quotidienne dans mes projets de recherche, c'est un chantier encore largement inexploré, alors, on essaye des formes, des visualisations, et on teste sur le public! Prenons l'exemple des aéroports et des processus de marchandisation en cours. Quand j'arrive dans un terminal d'aéroport, quand je constate que je suis obligé de passer par le duty-free pour rejoindre la porte d'embarquement de mon vol, je le ressens comme une forme de violence, une forme de confiscation de l'espace public auquel je peux prétendre. Je me pose cette question pour toutes les formes de violence (sociales, économiques, symbolique, institutionnelles, etc.). C'est pour analyser ces processus de confiscation de nos espaces de vie - voire de nos prérogatives de citoyens - que je crois nécessaire de produire une cartographie à toutes les échelles.

Par la cartographie expérimentale, on peut se faire témoin de situations où le cartographe a ressenti la violence du monde. Et c'est l'outil idéal pour pouvoir représenter cette indignation. Trevor Paglen, par exemple, qui n'était qu'un citoyen engagé qui a utilisé la cartographie pour dénoncer une situation qui l'indignait (il a en outre cartographier à l'aide d'un réseau participatifs les vols secrets de la CIA qui transportait les prisonniers durant la guerre en Afghanistan) ${ }^{10}$.

La terminologie et la manière dont on nomme les choses sur la carte peut aussi être l'expression d'une forme de violence, un moyen d'imposer son pouvoir ou de nier l'existence de "l'autre " [Rekacewicz, 2006d, 2006e et 2006f]. Cela suscite une aussi une colère, encore faut-il pouvoir l'exprimer. Dans les années 1990, les projets de Franjo Tuđman ou de Slobodan Milošević faisaient disparaître les Bosniaques, par un partage de la BosnieHerzégovine entre la Croatie et la Serbie. Ces projets ont été formalisés sur des cartes : je pense à l'esquisse réalisée par Tuđman en 1995, dessinée sur la nappe en papier d'un restaurant pour un journaliste anglais à Londres, esquisse sur laquelle Tudjman avait simplement oublié de représenter les Bosniaques). En Israël-Palestine, parler d'un « quartier juif " de Jérusalem-Est n'a pas la même résonnance politique que de parler de "colonie israélienne illégale aux termes de la résolution 242 de l'ONU». Or, sur les cartes

\footnotetext{
${ }^{10}$ A ce propos, voir : "Mapping Ghosts (excerpt) | Visible Collective \& Trevor Paglen », site An Atlas Radical Cartography, en ligne : http://www.an-atlas.com/contents/pag_em_vis.html
} 
israéliennes, les colonies sont appelées "villes ». Autre exemple, la ville palestinienne de Hébron (environ 200000 habitants) disparaît de certaines cartes israéliennes lesquelles figurent pourtant la colonie israélienne immédiatement voisine de Kyriat Arba (7000 habitants, essentiellement des colons). C'est une sorte «nettoyage ethnique cartographique ". C'est la carte qui fait disparaître les peuples qui restent pourtant sur le terrain, c'est la carte qui dicte une vision du monde, au sens de Carl Ritter. On pourrait aussi évoquer les conflits autour de la nomination des mers et de certaines régions (Mer du Japon ou Mer de l'Est, Golfe Persique, macédoine, etc.) [voir : Rekacewicz, 2006d, 2006e et 2006f]. Ce débat terminologique est le premier qui suscite des émotions: la manière dont on représente un pays, dont on nomme un lieu, est un acte très émotionnel pour les gens, qui peuvent ressentir l'utilisation de certains toponymes, de certaines représentations géographiques comme des insultes. [voir : Courrier international, 2008]. II y a, autour de ces cartes, une crispation historique extrêmement sensible, Doit-on écrire sur la carte "golfe persique ", "golfe arabo-persique ", "golfe " tout court ? Doit-on considérer sérieusement le conflit toponymique entre la Grèce et la Macédoine : un embargo grec a même été mené contre la Macédoine, jusqu'à ce que les macédoniens acceptent - du bout des lèvres - que le nom officiel deviennent ARYM [Ancienne République yougoslave de Macédoine, FYROM en anglais - Former Yugoslav Republic of Macedonia] pour plaire aux autorités grecques qui revendiquent le nom de Macédoine. Tant de décisions politiques se prennent sur des "frustrations d'empire »: la Grèce, l'Albanie, la Serbie, la Turquie, l'Arménie, etc. Cela constitue un chantier phénoménal pour la cartographie! Nommer les territoires et représenter la toponymie ${ }^{11}$, c'est faire référence à une vision, à une approche extrêmement émotionnelle de l'espace, souvent produite par des traumatismes historiques.

BT : Finalement, doit-on passer par la carte pour parler des émotions ? La carte n'est-elle pas elle aussi un filtre qui empêche, au moins partiellement, de dire les émotions ?

PR : Reclus voulait faire de la cartographie sans la carte. C'est l'un des premiers à expliquer que le monde est un grand système. En cela, c'est le père de la géographie systémique. Or, il détestait les cartes de son époque, car elles étaient figées et purement descriptives [voir : Ferretti, 2007 ; Collignon, 2007]. D'où son travail sur la cartographie que Charles Perron avait entreprit en collaboration, avec Reclus, pour laquelle ils voulaient penser les interactions, les systèmes, avant même de représenter le monde. Nous avons bien sûr rebondi sur cette idée : la carte qui pourrait s'émanciper du fond de carte.

C'est l'idée qui sous-tend le projet de " la grande roue africaine " (Figure 7) et de celle de "I'histoire du monde en cinq actes et un entre acte ". La " machinerie ", ou les " rouages africains " privilégient la représentation des liens qui unissent l'Afrique et ses principaux partenaires dans un échange bijectif et complexe. Le fond de carte n'est ici qu'un alibi, ce qui est important, c'est la narration, la circulation des objets de la géographie entre l'Afrique et les autres continents La série historique réalisée en cinq tableaux (et un entre acte) montre qu'on peut faire du spectacle avec la représentation cartographique et rappelle que les cartes ne sont pas la réalité, qu'elles sont un subtil dialogue entre l'imaginaire et la réalité, comme une pièce de théâtre basée sur des faits réels, mais romancée pour les besoins de la

\footnotetext{
${ }^{11}$ A ce propos, on pourra se reporter au carnet de recherche Neotopo (toponomascapes en construction, étude en toponymie politique) : https://neotopo.hypotheses.org
} 
cause. II y a des décors, des acteurs, des paysages, des histoires qu'on raconte à partir de faits réels réinterprétés. Dans ce projet, il n'y a pas l'ombre d'un fond géographique. Toute I'histoire est racontée à l'aide de symboles abstraits, des ronds, des ovales ou des rectangles qui sont autant d'évocation des pays ou des continents. Une manière de se défaire du fond de carte, de privilégier la représentation des processus et des changes plutôt que des formes géographique des continents. Cela permet un découplage entre la carte et ce que dit la carte.

Figure 7 : La " grande roue africaine » : les échanges euro-africains

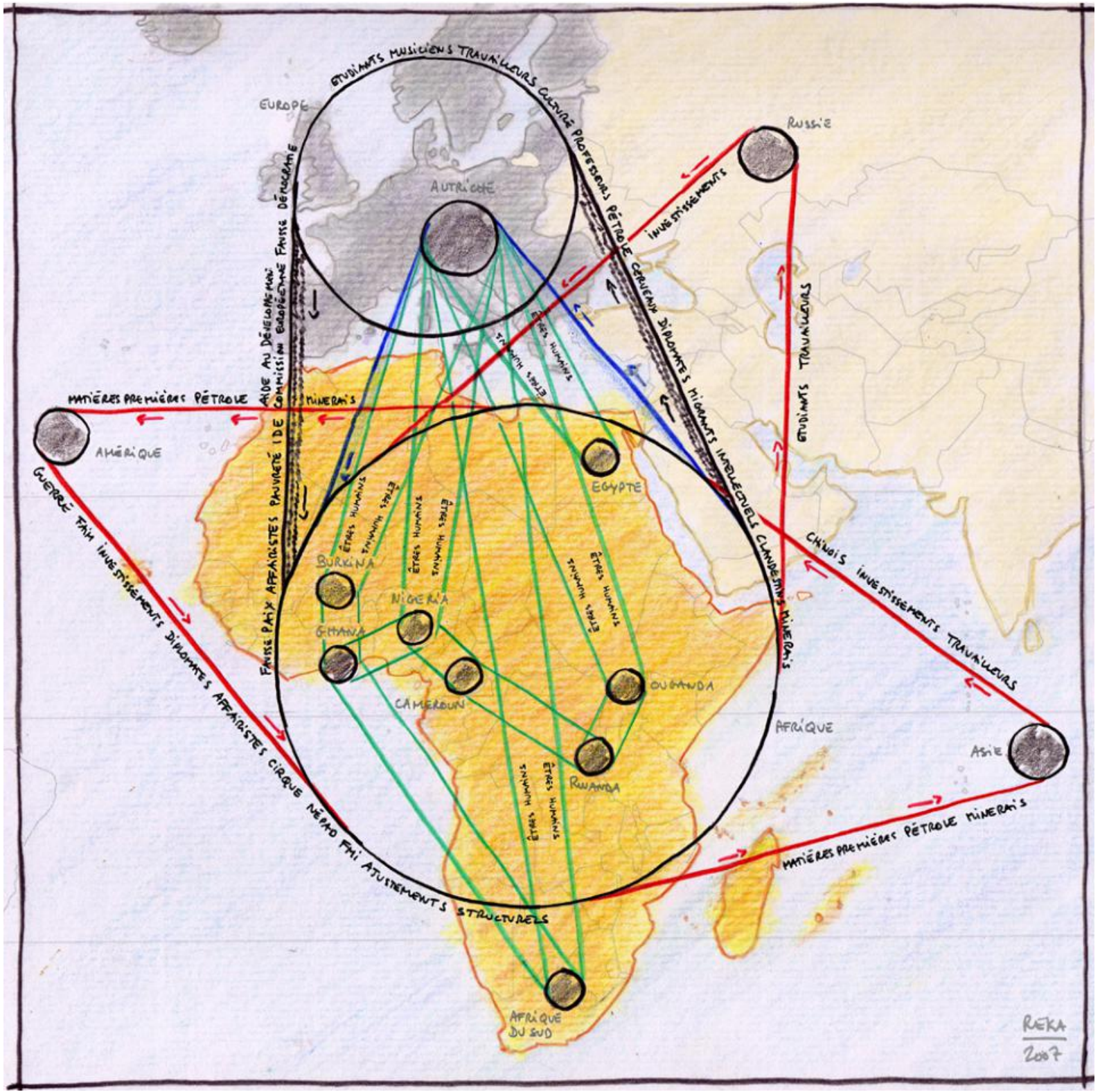

Source : Rekacewicz, 2007a.

Le monde contemporain est marqué par l'espace virtuel et l'hypermobilité. L'économie est encore profondément ancrée dans l'espace (usine, transports), mais est aussi en partie dématérialisée (paradis fiscaux, flux financiers). C'est de là qu'est née l'idée de s'émanciper de la carte pour faire de la cartographie, et se concentrer sur l'étude des systèmes, des processus. Ce discours est un peu provocateur: l'idée est de dire qu'on peut être plus 
efficace en montrant l'immatérialité. Ce qui m'amène à engager parallèlement une recherche en design graphique. Une sorte de défi : transformer le savoir, les informations, les processus humains en formes graphiques utilisant, par exemple, l'art abstrait pour en produire une retranscription visuelle. Pour aborder cet aspect de mes recherches, Je me suis plongé dans les oeuvres des artistes du Bauhaus, de Kandinsky, Klee, un peu Picasso, Jasper Johns, des artistes russes comme Lissitzky ou Sternberg. Mais il faut que ce symbolisme reste accessible.

BT : Revenons pour finir aux liens entre arts, cartographie et émotions. Est-ce parce qu'elle s'inspire des pratiques de la carte par les artistes que la cartographie expérimentale, que vous décrivez comme " une forme de contestation au service de la justice sociale" (Rekacewicz, 2013d), peut si bien appréhender et rendre les émotions ?

PR : Pour moi, l'art c'est avant tout de l'émotion. Et la cartographie est résolument une œuvre artistique, une illustration, peu importe comment on l'appelle d'ailleurs. C'est pour cela qu'il est souvent question des liens entre l'art et la cartographie sur visionscarto.net.

La cartographie est donc une discipline qui emprunte à l'art, et il faut simplement qu'elle soit pertinente. La cartographie entretient notamment une relation très intime avec le travail $d^{\prime}$ esquisse. Non sans raisons : le monde est en mouvement et l'esquisse permet une approche modeste. C'est une réhabilitation de l'imprécision, qui fâche les cartographes orthodoxes. En réalité, on a beaucoup de mal à inventer de nouveaux modèles cartographiques. Parce que nous sommes habitués à ces flux, ces flèches, ces ronds, qui structurent notre pensée. Et les très rares artistes ou activistes qui ne font pas du tout référence à cette sémiologie graphique nous décontenancent totalement. Face à certaines ceuvres cartographiques, il arrive que j'éprouve des émotions, sans pour autant en comprendre le sens. Aussi, j'ai préféré mixer les éléments de la cartographie orthodoxe à ma propre cartographie radicale, quitte à faire évoluer le tout. (Rekacewicz, dans Volcler, Zortea, 2014).

L'art est une source inépuisable d'inspiration pour la cartographie, pour inventer des formes, des atmosphères. Je garde en mémoire ce que m'a appris Michel Morel, qui emmenait ses étudiants dans des musées de l'art, et qui nous disait que c'était là que la cartographie commençait, dans ces musées [voir: Rekacewicz, 2014c]. Je suis très influencé par l'art abstrait, l'école du Bauhaus, et des artistes comme Klee, Popova ou Kandinsky (qui est celui qui, en 1926, a publié en allemand le livre qui a conceptualisé les fondements de la cartographie : Points et lignes sur plan), Feininger, mais aussi Albert Gleizes et Abdoulaye Konaté (pour leur approche inimitable des jeux de couleur).

Edward Hopper m'a fortement inspiré également, par sa mise en scène des paysages en les dramatisant, notamment par le jeu des ombres, d'où l'idée d'intégrer du relief (et non pas de la 3D !) dans la carte. L'influence artistique, la source d'inspiration, c'est aussi notre sensibilité au paysage. Pendant longtemps, je me suis interrogé sur la forme que pouvait prendre un des objets les plus difficiles à représenter en cartographie : la flèche. C'est un paysage qui m'a donné la réponse. Je me baladais en Bretagne, aux alentours de la baie des Trépassés, dans laquelle les vagues arrivaient en " rideau ». Leur forme était magnifique, les courbes élégantes : j'ai pris beaucoup de photographies, depuis les collines. Et en rentrant à l'atelier, j'ai essayé d'appliquer sur les cartes les formes courbes des vagues que j'avais observées. Ces formes et ces mouvements peuplent ma cartographie depuis plus de deux décennies. Les formes et les couleurs de la nature, tout comme les formes architecturales, sont autant de sources d'inspirations pour le cartographe qui veut transmettre des émotions 
par la carte. La nature, souvent, nous suggère les solutions graphiques à des problèmes cartographiques insolubles.

BT : Sur ces liens entre arts, émotions et cartographie, il ne me reste qu'à vous remercier.

PR : Merci pour m'avoir permis de présenter des émotions dans la cartographie critique et expérimentale.

\section{Bibliographie indicative}

Casanova V., Izambert C. (2010), « La carte, un objet graphique. Entretien avec Philippe Rekacewicz", Vacarme, 21 janvier 2010, en ligne: http://www.vacarme.org/article1847.html

Collignon B., Staszak J.-F. (dir.), 2003, Espaces domestiques. Construire, habiter, représenter, Paris, Bréal, 447 p.

Collignon B. (2007), "Elisée Reclus et la cartographie (2/2). Le monde sans la carte ", Visions cartographiques, 13 novembre 2007, en ligne : http://blog.mondediplo.net/2007-11-13-Lemonde-sans-la-carte

Comité international de Bologne pour la cartographie et l'analyse du monde contemporain, 2008, "Philippe Rekacewicz, un cartographe confronté à la représentation des frontières ", Géoconfluences, 11 mars 2008, en ligne: http://geoconfluences.enslyon.fr/doc/typespace/frontier/FrontViv.htm

Courrier international, 2008, L'Atlas des atlas. Le monde vu d'ailleurs en 200 cartes, Paris, Arthaud, $191 \mathrm{p}$.

Grataloup C., Salachas J., Sarazin J.-Y. (2013), "L'âge d'or des cartes marines. Quand l'Europe découvrait le Monde ", Cafés géographiques, rubrique Des Cafés, compte-rendu du café géo-expo du 12 janvier 2013 par B. Tratnjek, en ligne: http://cafegeo.net/article_2508/

Olmedo E. (2011), "Cartographie sensible, émotions et imaginaire ", Visions cartographiques, 19 septembre 2011, en ligne: http://blog.mondediplo.net/2011-09-19Cartographie-sensible-emotions-et-imaginaire, article republié le 15 mars 2015 : http://visionscarto.net/cartographie-sensible

Olmedo E., Poisson M. (2012), "Cartographier les interstices de la ville ", Strabic, 8 mai 2012, en ligne : http://strabic.fr/Mathias-Poisson-Cartographier-les-interstices-de-la-ville

Olmedo E., Roux J.-M. (2014a), "Conceptualité et sensibilité dans la carte sensible (1/2) », Implications Philosophiques, 31 mars 2014, en ligne: http://www.implicationsphilosophiques.org/actualite/une/conceptualite-et-sensibilite-dans-la-carte-sensible-12/

Olmedo E., Roux J.-M. (2014b), "Conceptualité et sensibilité dans la carte sensible (2/2) », Implications Philosophiques, $1^{\text {er }}$ avril 2014, en ligne: http://www.implicationsphilosophiques.org/actualite/une/conceptualite-et-sensibilite-dans-la-carte-sensible-22/

Olmedo E. (2015a), « Représenter l'espace vécu. Les enjeux d'une cartographie émotionnelle en géographie ", séminaire Géographie des émotions, ENS-Ulm, 15 janvier 2015, compterendu par Célia Innocenti et Aurore Léon, en ligne: http://geographie.ens.fr/IMG/file/Géo\%20des\%20émotions/Geoemotions_CR\%2015\%20janvier.pdf 
Olmedo E. (2015b), Cartographie sensible. Tracer une géographie du vécu par la rechercheaction, thèse de doctorat en géographie, Université Paris 1 Panthéon-Sorbonne.

Feidel B., Olmedo E., Troin F. Depeau S., Poisson M., Audas N., Jaulin A., Duplan K. (2016), "Parcours augmentés, une expérience sensible entre arts et sciences sociales ", Carnets de géographes, $n^{\circ} 9$, rubrique Carnets de terrain.

Ferretti F. (2007), "Elisée Reclus et la cartographie (1/2). Élisée Reclus, le géographe qui n'aimait pas les cartes ", Visions cartographiques, 13 novembre 2007, en ligne: http://blog.mondediplo.net/2007-11-11-Elisee-Reclus-le-geographe-qui-n-aimait-pas-les

Papin D. (2012), "L'évolution de la place de la cartographie dans le journal Le Monde ", Hérodote, n¹46-147, pp. 108-118, en ligne : http://www.cairn.info/revue-herodote-2012-3page-108.htm

Rekacewicz P. (2001a), "Haut-Karabagh: projet d'échange de territoires (Swap) ", Le Monde diplomatique, mars 2001, en ligne: https://www.mondediplomatique.fr/2001/03/REKACEWICZ/46650

Rekacewicz P. (2006a), "Naissance d'une carte ", Visions cartographiques, 9 novembre 2006, en ligne : http://blog.mondediplo.net/2006-11-09-Naissance-d-une-carte

Rekacewicz P. (2006b), "Le partage des Mondes ", Visions cartographiques, 9 novembre 2006, en ligne : http://blog.mondediplo.net/2006-11-09-Le-partage-des-Mondes

Rekacewicz P. (2006c), "L'Europe et ses frontières paradoxales », Visions cartographiques, 27 novembre 2006, en ligne: http://blog.mondediplo.net/2006-11-27-L-Europe-et-sesfrontieres-paradoxales

Rekacewicz P. (2006d), " Nommer c'est exister! ", Visions cartographiques, 19 décembre 2006, en ligne : http://blog.mondediplo.net/2006-12-19-Nommer-c-est-exister

Rekacewicz P. (2006e), " Nommer c'est exister! (2) ", Visions cartographiques, 21 décembre 2006, en ligne : http://blog.mondediplo.net/2006-12-21-Nommer-c-est-exister-2

Rekacewicz P. (2006f), " Nommer c'est exister! (3) ", Visions cartographiques, 23 décembre 2006, en ligne : http://blog.mondediplo.net/2006-12-21-Nommer-c-est-exister-3

Rekacewicz P. (2006g), "La cartographie, entre science, art et manipulation », Atlas 2006 du Monde diplomatique, en ligne : https://www.monde-diplomatique.fr/cartes/manipulation

Rekacewicz P. (2007a), "Rendez-vous à Sharon's Stone», Visions cartographiques, 7 novembre 2007, article republié en février 2016, en ligne : http://visionscarto.net/rendezvous-a-sharon-stone

Rekacewicz P. (2007b), "Migrations et réfugiés: le monde qui accueille et celui qui se ferme ", Visions cartographiques, 5 avril 2007, en ligne : http://blog.mondediplo.net/200704-04-Migrations-et-refugies-le-monde-qui-accueille-et

Rekacewicz P ; (2009), " Migrations, sauvetage et droits humains », Visions cartographiques, 27 septembre 2009, en ligne : http://visionscarto.net/migrations-sauvetage-en-mer

Rekacewicz P. (2013a), " Cartes en colère ", Visions cartographiques, 22 décembre 2013, en ligne : http://blog.mondediplo.net/2013-12-22-Cartes-en-colere

Rekacewicz P. (2013b), "Aéroports, de l'espace public à l'espace privé », Le Monde diplomatique, février 2013, pp. 13-16, en ligne: https://www.mondediplomatique.fr/2013/02/REKACEWICZ/48733

Rekacewicz P. (2013d), "Cartographie radicale ", Le Monde diplomatique, février 2013, p. 15, en ligne: http://www.monde-diplomatique.fr/2013/02/REKACEWICZ/48734

Rekacewicz P. (2014a), "Mourir aux portes de l'Europe ", Visions cartographiques, 28 avril 2014, en ligne : http://visionscarto.net/mourir-aux-portes-de-l-europe 
Rekacewicz p. (2014b), "La Méditerranée, plus loin que I'horizon », Visions cartographiques, 6 mai 2014, en ligne : http://visionscarto.net/la-mediterranee-plus-loin

Rekacewicz P. (2014c), "Dans les musées de Vienne, la mort et la vie ", Visions cartographiques, 23 décembre 2014, en ligne : http://visionscarto.net/dans-les-musees-devienne

Staszak J.-F. (dir.) (2001), "Espaces domestiques ", Annales de Géographie, vol. 110, n620, en ligne : http://www.persee.fr/issue/geo_0003-4010_2001_num_110_620

Volcler J., Zortea J. (2014), "Dessiner une carte avec trois orangers ", Article 11, n¹8, en ligne : http://www.article11.info/?Dessiner-une-carte-avec-trois

\section{Sources des cartes}

Clochard O., Rekacewicz P. (2006), "Des morts par milliers aux portes de l'Europe ", Le Monde diplomatique, mars 2004, version 2006 en ligne: https://www.mondediplomatique.fr/cartes/mortsauxfrontieres

Rekacewicz P. (2001b), "Haut-Karabagh: le grand swap n'aura pas lieu », Le Monde diplomatique, mars 2001, en ligne: https://www.mondediplomatique.fr/cartes/armenieazerbaidjan

Rekacewicz P. (2010), " Guerres et armements », Le Monde diplomatique, octobre 2010, en ligne : https://www.monde-diplomatique.fr/cartes/armementsconflits

Rekacewicz P. (2012), "L'arc des réfugiés ", Le Monde diplomatique, juin 2012, en ligne: https://www.monde-diplomatique.fr/cartes/arcdesrefugies

Rekacewicz P. (2013c), "L'aéroport à l'assaut des passagers ", Le Monde diplomatique, février 2013, pp. 13-16, https://www.monde-diplomatique.fr/cartes/dutyfree

Rekacewicz P. (2015), "Qui fabrique les armes, et qui les achète? ", Visions cartographiques, en ligne : http://visionscarto.net/qui-fabrique-les-armes-et-qui-les-achete 